\title{
Evaluación rápida de las familias y grupos tróficos de aves asociadas a sabanas y bosques de galería en la Reserva El Caduceo, La María (San Martín, Meta, Colombia)
}

\author{
Rapid Assessment of the Families and Groups of Trophic Birds \\ Associated with Savannas and Gallery Forests at El Caduceo \\ Nature Reserve, La María (San Martín, Meta, Colombia)
}

\section{Luis Alejandro Yañez-Dukon ${ }^{\text {ab }}$, Nixon Fernando Vargas Hernández ${ }^{\mathrm{a}}$, Isabela Forero Espinosa a , Pablo Locano Montoya ${ }^{a}$, Jordan Steven Ruiz Toquica ${ }^{a c}$}

\footnotetext{
a Programa de Biología Marina, Facultad de Ciencias e Ingenierías, Universidad de Bogotá Jorge Tadeo Lozano, Colombia

b luisa.yanezd@utadeo.edu.co | https://orcid.org/0000-0002-6141-0412

c jordan.ruiz@utadeo.edu.co| https://orcid.org/0000-0002-5456-2434
}

\section{RESUMEN}

La Reserva El Caduceo, ubicada en San Martín, Meta, es un área que ha experimentado notables modificaciones en la complejidad estructural de la vegetación de bosques y sabanas debido al efecto de diferentes actividades de cultivo y ganadería, las cuales pueden afectar la riqueza de las comunidades de aves que allí habitan. Este estu-

Citation: Yañez-Dukon, L. A., Vargas-Hernández, N. F., Forero-Espinosa, I, Locano-Montoya, P. y Ruiz-Toquica, J. S. (2021). Evaluación rápida de las familias y grupos trófico de aves asociadas a sabanas y bosques de galería en la Reserva El Caduceo, La María (San Martín, Meta, Colombia). Mutis, $11(2), 132-142$. https://doi.org/10.21789/22561498.1705

Recibido: 16 de diciembre de 2020. Aceptado: 30 de marzo de 2021

Copyright: @2021. Yañez-Dukon, L. A., Vargas-Hernández, N. F., ForeroEspinosa, I., Locano-Montoya, P. y Ruiz-Toquica, J. S. (2021). This is an open-access article, which permits unrestricted use, distributions and reproduction in any medium, provided the original author and source are credited.

Competing Interests: The authors have no conflict of interest. dio corto muestra una evaluación de la composición y abundancia a nivel de familias y grupos tróficos de aves en esta reserva durante la época lluviosa de 2020. Se llevaron a cabo censos visuales con límite de tiempo en dos estaciones correspondientes a dos hábitats: bosque de galería y sabana con pequeños núcleos de bosque. Además, se estimaron algunos índices ecológicos para cada estación de muestreo. Se observó un total de 18 familias, de las cuales la familia Tyrannidae fue la más abundante en la sabana y la familia Psittacidae la más recurrente en el bosque de galería. La densidad de aves fue mayor en el bosque de galería $\left(2,19 \mathrm{ind} / \mathrm{m}^{2}\right)$, observando además que en esta estación las densidades de 
aves se encuentran equitativamente repartidas entre las familias registradas, en contraste con la estación de sabana. Por otro lado, el grupo trófico dominante en ambas estaciones fueron los "insectívoros". Estos resultados preliminares podrían indicar una relación directa entre la complejidad estructural vegetativa, la recuperación del bosque de galería en esta zona y la composición de aves y grupos tróficos predominantes. No obstante, futuros estudios con mayor duración y monitoreos constantes son necesarios para confirmar esta observación inicial.

Palabras clave: avifauna, sabanas, bosques de galería, grupo trófico.

\section{ABSTRACT}

El Caduceo Nature Reserve, located in San Martín, Meta, is an area that has undergone notable modifications in the structural complexity of the vegetation of its forests and savannas due to different farming and livestock activities, which could affect the richness of resident bird communities. This brief study shows an evaluation of the composition and abundance at the level of families and trophic groups of birds in this reserve during the rainy season of 2020. Visual censuses with a time limit were carried out in two stations corresponding to two habitats: gallery forest and savanna with small core forest. Some ecological indices were estimated for each sampling station as well. A total of 18 families were observed, with the Tyrannidae family being more abundant in the savannah, while the Psittacidae family in the gallery forest. The density of birds was higher in the gallery forest $\left(2.19 \mathrm{ind} / \mathrm{m}^{2}\right)$, station in which bird densities are equitably distributed among the recorded families, in contrast to what occurs in the savanna station. On the other hand, the dominant trophic group in both stations were "insectivores." These preliminary results could indicate a direct relationship between the vegetative structural complexity, the recovery of the gallery forest in this area, and the composition of birds and predominant trophic groups. However, future studies with longer duration and constant monitoring are necessary in order to confirm this introductory observation.

Keywords: Avifauna, savannas, gallery forests, trophic group.

\section{INTRODUCCIÓN}

Colombia es considerado un país megadiverso y de alto valor para la conservación (Fonseca \& Mahecha, 2018), siendo reconocido por la alta diversidad de aves que alberga, pues cuenta con aproximadamente 1.909 especies, ocupando el primer lugar a nivel global en este grupo (Asociación Colombiana de Ornitología, 2020). Entre las regiones más diversas del país, la Orinoquía se destaca por la riqueza de aves, ya que en esta se han registrado cerca de 761 especies, es decir, aproximadamente $19 \%$ de las especies a nivel mundial y $47,6 \%$ de las especies registradas para el país (Tamaris-Turizo et al., 2017), las cuales están representadas por 106 familias, entre las que se encuentran las familias Tyrannidae, Thamnophilidae, Thraupidae, Furnariidae y Trochilidae, las más representativas de la región (Acevedo-Charry et al., 2014). Tal diversidad se encuentra concentrada en los ecosistemas de altillanuras, sabanas inundables, bosques de galería, bosques subandinos y bosques del escudo Guyanés, entre otros, típicos de esta región (Peñuela et al., 2011).
La Orinoquía ha sufrido notables modificaciones paisajísticas a causa de diferentes actividades antropogénicas tales como la ganadería, la expansión de la frontera agrícola y plantaciones de palma africana, lo cual ha alterado la composición y complejidad estructural de la vegetación de tal forma que sus paisajes se están volviendo monótonos, lo que a su vez genera un impacto sobre la diversidad de aves que alberga esta región (a mayor complejidad, mayor diversidad de aves) (Huertas-Ramírez \& Huertas-Herrera, 2015; Peñuela et al., 2011; Tamaris-Turizo et al., 2017). Un ejemplo de ello es la zona de San Martín, en el departamento del Meta, específicamente en la Reserva el Caduceo, donde ha ocurrido una sucesión ecológica del bosque debido a la ganadería extensiva y los cultivos de palma de aceite, actividades que han generado cambios en la composición y estructura de la vegetación (Díaz, 2016; Tamaris-Turizo et al., 2017).

Las aves desempeñan un papel ecológico importante en estos ecosistemas boscosos dada su alta interacción directa e indirecta con las comunidades forestales (Winkler \& Preleuthner, 2001). Además, gracias a 
sus dietas, estos organismos ayudan a determinar la estructura y organización de las comunidades, bien sea regulando procesos de flujo de energía o interviniendo en el intercambio genético de la vegetación al ser dispersores de semillas (González-Salazar et al., 2014; Olsen, 2017; Taylor et al., 2017), ocupando así diferentes grupos tróficos, que son distinguibles por las diversas formas de sus picos (González-Salazar et al., 2014; Martínez et al., 2010). A pesar de esta importancia, existen pocos registros actuales sobre la diversidad de aves asociadas a San Martín, Meta. Los estudios más recientes de aves en el municipio fueron realizados por Tamaris-Turizo et al. (2017), además de un registro fotográfico de la composición de aves a nivel de familia en la reserva El Caduceo realizado por Melo-Velasquez y Vega-Araque (2019).

El presente estudio muestra una aproximación a la composición de aves a nivel de familia y de grupos tróficos en sabanas y bosques de galería de la Reserva El Caduceo, San Martín (Meta, Colombia). Para ello, se evaluó la estructura (composición y abundancia a nivel de familia) de dos comunidades de aves a través de censos visuales. Así mismo, se evaluó la estructura de los grupos tróficos de la zona con base en lo reportado en la literatura.

\section{METODOLOGÍA}

\section{Área de estudio}

El estudio se llevó a cabo los días 14 y 15 de noviembre de 2020 en la Reserva El Caduceo, ubicada en el área rural de San Martín, departamento del Meta, Colombia (figura 1a), la cual tiene una elevación de 377,8 m s. n. m. (Fonseca \& Mahecha, 2018) y se localiza entre los 0340'17" N y los 7339'34" W, limitando al norte con los municipios de Guaymaral, Castilla La Nueva, San Carlos De Guaroa y Puerto López, al sur con Fuentedeoro, Puerto Lleras y Mapiripán, al oeste con Granada, El Castillo, El Dorado y Cubarral, y al este con Puerto Gaitán (León \& Mojica, 2018). La temperatura promedio anual en esta zona es de $26 \stackrel{\circ}{\circ}$, con oscilaciones entre 23,1 y 28,2 으, mientras que las precipitaciones promedio anuales son de 255,4 $\mathrm{mm}$, con regímenes de lluvia que se extienden desde el mes de abril hasta noviembre y períodos secos que van desde diciembre hasta marzo (Cantillo, 2009; Fonseca \& Mahecha, 2018).
La reserva cuenta con un área de 173 ha, de las cuales el $60 \%$ de la vegetación corresponde a sabanas naturales y pastos introducidos, mientras que el $40 \%$ restante corresponde a bosque de galería y pequeños núcleos de bosque aislados en la sabana, como matas de monte y arbustales (Casallas-Pabón et al., 2017). Las especies vegetales dominantes de esta zona corresponden a Euterpe precatoria e Iriartea deltoidea (Bosque de vega), Vismia mocanera, Dicanopteris sp. (brizal), Vismia sp., Solanum quitoense y Sepium sp. (vegetación pionera), Mauritia flexuosa, Matayba, Jacarda y otras de la familia Melastomatácea. En bosque secundario se encuentra Sclerobium y Virola (León \& Mojica, 2018). Una gran parte de los bosques de galería se encuentran asociados a varios cuerpos de agua, entre ellos, arroyos, ríos y caños (León \& Mojica, 2018), de los cuales el caño Camoa atraviesa el área de estudio al norte del campamento, como se muestra en la figura 1.

Figura 1. Área de estudio

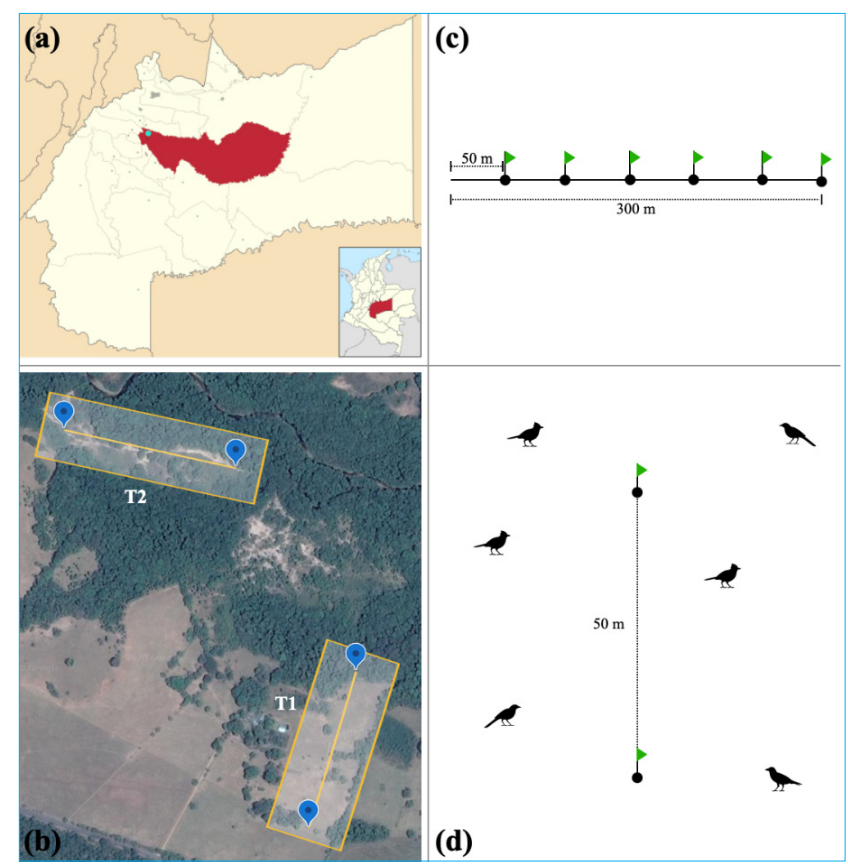

(a) ubicación de la Reserva El Caduceo, La María, San Martín (Meta, Colombia); (b) ubicación de las estaciones 1 (T1) y 2 (T2) dentro de la reserva; (c) disposición de los puntos de muestreo dentro del transecto de $300 \mathrm{~m}$; (d) recuento en el punto dentro del transecto sin estimación de distancia.

Fuente: adaptado de Wikipedia (2012) y Google Maps (2020). 


\section{Muestreo}

Se siguió la metodología propuesta por Gallina y López-Gonzáles (2011), con algunos ajustes. La selección de los transectos dentro del área de estudio se basó en los criterios propuestos por Moreno (2017), con algunas modificaciones, entre las cuales se incluyeron: constituir una unidad discreta de paisaje (sabanas y bosque de galería, respectivamente) y ubicar el transecto en una altitud y pendiente de interés. Para ello, se dispusieron dos estaciones de muestreo: la estación 1 (T1, 339'23" N, 7339'23"

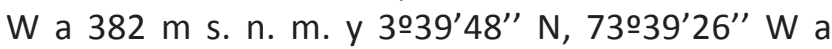
$374 \mathrm{~m} \mathrm{~s}$. n. m.), representada por una unidad paisajística de sabanas y pequeños núcleos de bosques de galería (figura 1b); y la estación 2 (T2, 3 40'09" $\mathrm{N}, 73 \circ 39^{\prime} 30^{\prime \prime} \mathrm{W}$ a $400 \mathrm{~m}$ s. n. m. y 0340'11.6" N, 07339'40" W a 359 m s. n. m.), representada por bosques de galería asociados al caño Camoa (figura 1b). En cada estación se marcó un transecto de $300 \mathrm{~m}$, señalando seis puntos de conteo separados a una distancia de $50 \mathrm{~m}$ entre sí (figura 2c). En cada punto se realizó un censo visual durante $10 \mathrm{~min}$, con recuentos sin estimación de distancia (MacGregorFors et al., 2010; González-García, 2011) (figura 2d). El área total de muestreo de cada estación fue de $39,302 \mathrm{~m}^{2}$, con un perímetro de $942,65 \mathrm{~m}$.

Las observaciones de aves se realizaron en dos momentos del día para cada transecto, en la mañana entre $6: 00$ y 7:00 a. m. y en la tarde entre $5: 00$ y 6:00 p. m., utilizando binoculares $8 \times 40$. Los avistamientos se registraron teniendo en cuenta las indicaciones de Ralph et al. (1996), es decir, fecha, zona, observador, número de visita, transecto, punto de conteo, hora de muestreo, familia de aves observadas y número de individuos visualizados.

La identificación de aves a nivel de familia se llevó a cabo siguiendo la guía fotográfica de Melo-Velasquez y Vega-Araque (2019). Luego de haber identificado las familias, se hizo una búsqueda en la literatura sobre cada grupo con base en la dieta típica de las aves reconocidas, esto con el fin de observar los grupos tróficos que se pueden estar dando dentro de la comunidad de avifauna.

\section{Análisis de datos}

Se calcularon la densidad promedio y la abundancia relativa. Los índices de dominancia de Simpson $(\lambda)$, equitatividad de Pielou $(J)$ y similitud de jaccard $(I J)$ se hallaron mediante las siguientes fórmulas, tomadas de Ramírez (2006):

Índice de dominancia de Simpson $(\lambda)$

$$
\lambda=\sum_{i=1}^{S} p_{i}{ }^{2}
$$

Índice de equitatividad de Pielou (J)

$$
J=\frac{H^{\prime}}{\ln (S)}
$$

donde $S$ es el número de familias presentes, $N$ es el número total de individuos y pi es el número de individuos de la familia $i$.

Coeficiente de similitud de jaccard $\left(I_{J}\right)$

$$
I_{J}=\frac{c}{a+b-c}
$$

donde $a$ es el número de especies presentes en el sitio $A, b$ es el número de especies presentes en el sitio $B$ y $c$ es el número de especies presentes en ambos sitios, A y B.

\section{RESULTADOS}

Se registró un total de 136 individuos, distribuidos entre 18 familias de aves (anexo 1), de las cuales, las familias Falconidae, Galbulidae, Icteridae, Thraupidae y Tyrannidae estuvieron presentes en ambas estaciones. En T1 (estación 1, representada por la sabana) se observó un total de 11 familias, con una densidad total de $1,27 \mathrm{ind} / \mathrm{m}^{2}$. La familia Tyrannidae (24\%) fue la más abundante, seguida de Passerellidae (16\%) e Icteridae (14\%), mientras que las de menor abundancia fueron Thamnophilidae y Picidae (2 \%) (figura 2a). 
La comunidad evaluada mostró una dominancia $\lambda=$ 0,12 (anexos 2 y 3), con una equitatividad de $J=0,05$. Por su parte, en T2 (estación 2, dominada por el bosque de galería) se observó un total de 12 familias, con una densidad 2,19 ind $/ \mathrm{m}^{2}$, donde la familia Psittacidae $(40,7 \%)$ fue la más abundante, seguida de Galbulidae $(18,6 \%)$ y Falconidae $(15,1 \%)$. Las familias de menor abundancia fueron Tytiridae, Icteridae, Furnariidae, Corvidae y Ardeidae (1,2 \%) (figura 2b). En esta estación, la comunidad mostró una dominancia $\lambda=0,23$ (anexos 4 y 5 ) y una equitatividad $J=0,72$. En cuanto a la diversidad beta, es decir, entre las dos comunidades evaluadas, se obtuvo un $I_{J}=0,28$.

Figura 2. Algunas de las familias de aves identificadas en la Reserva El Caduceo, La María, San Martín (Meta, Colombia)

(a)
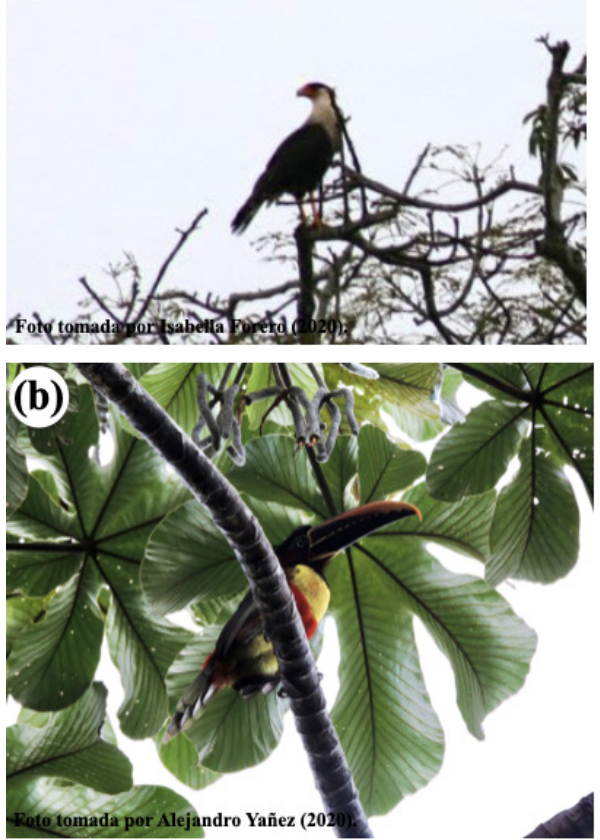

(c)

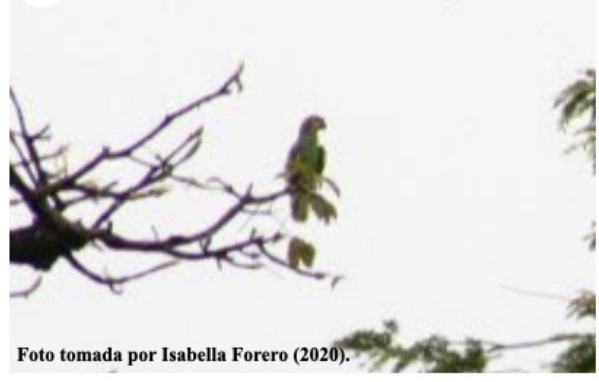

(d)

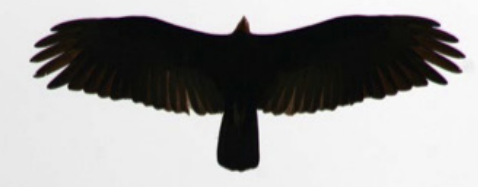

Foto tomada por Isabella Forero (2020).
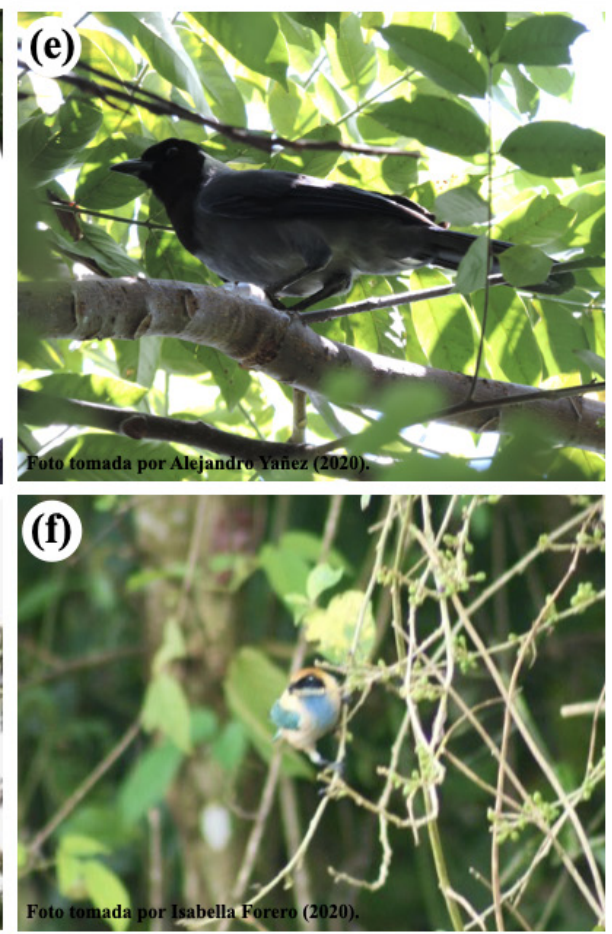

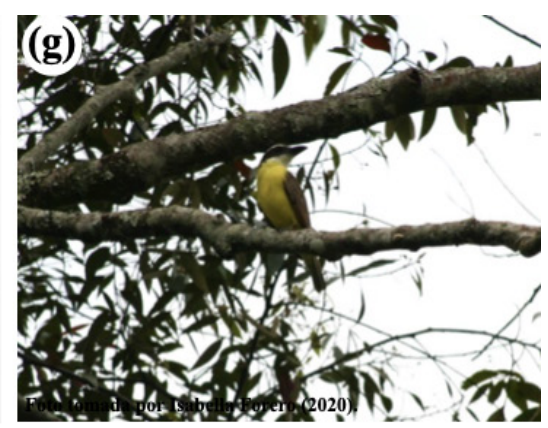

(h)

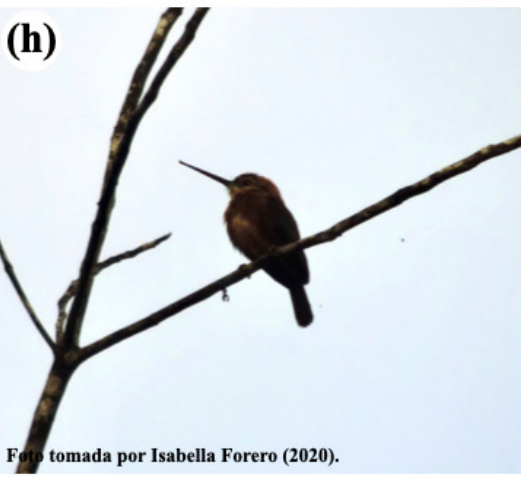

(i)

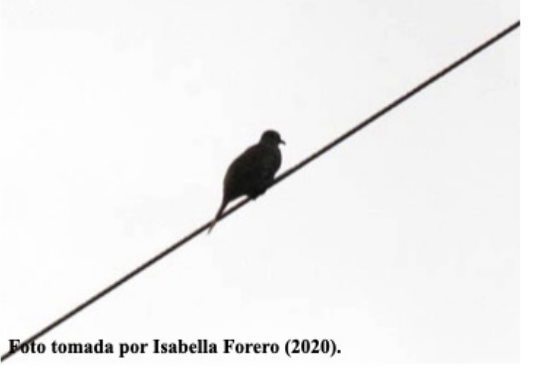

(a) Falconidae, (b) Ramphastidae, (c) Psittacidae, (d) Cathartidae, (e) Corvidae, (f) Thraupidae, (g) Tyrannidae, (h) Galbulidae y (i) Columbidae. 
En relación con la diversidad trófica, en T1 se identificaron ocho grupos tróficos, siendo el grupo insectívoro (36\% de las familias) el más representativo (figura 3), y el cual incluye a las familias Picidae, Tyrannidae, Trochilidae y Thraupidae. Las familias representadas por los grupos restantes se muestran en el anexo 1. En cuanto a T2, se identificaron cinco grupos tróficos, siendo los grupos frugívoro $(33,34 \%$ de las familias) e insectívoro (33,33 \%) los más representativos (figura 3). Estos grupos incluyeron las familias Corvidae, Psittacidae, Tersinidae y Tityridae (frugívoras) y Cuculidae, Furnariidae, Galbulidae y Tyrannidae (insectívoras). Las familias representadas por los grupos carnívoros, que tuvieron una menor representación, se detallan en el anexo 1.

Figura 3. Abundancia relativa y porcentajes de grupos tróficos de las aves registradas en la estación 1 (a) y la estación 2 (b) en la Reserva El Caduceo, La María, San Martín (Meta, Colombia)

(a)
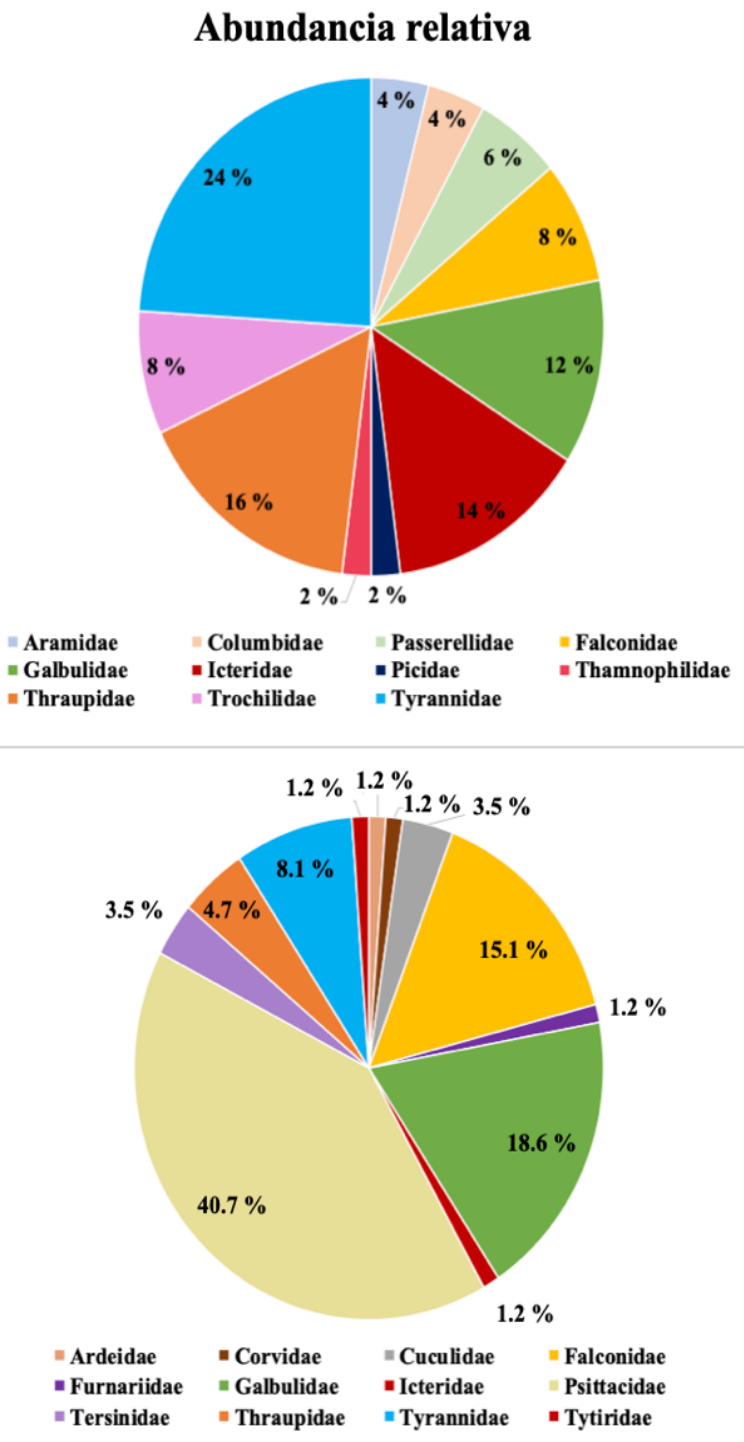

\section{(b)}

\section{Grupos tróficos}

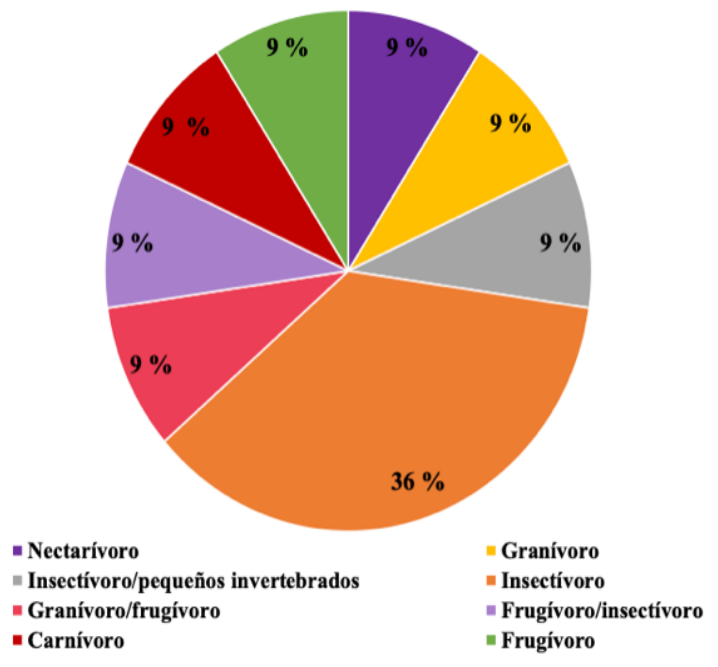

Fuente: elaboración propia. 
En cuanto a la densidad relativa, se observó que en T1 la familia Tyrannidae fue la más abundante $(0,305$ ind $\left./ \mathrm{m}^{2}\right)$, seguida de Thraupidae $\left(0,204 \mathrm{ind} / \mathrm{m}^{2}\right)$ e Icteridae $\left(0,178 \mathrm{ind} / \mathrm{m}^{2}\right)$. Las familias menos representadas fueron Thamnophilidae y Picidae, con densidades de $0,025 \mathrm{ind} / \mathrm{m}^{2}$ (figura 4 ). Por otro lado, en
T2 la familia Psittacidae fue la más abundante $(0,891$ ind $\left./ \mathrm{m}^{2}\right)$, seguida de Galbulidae $\left(0,407 \mathrm{ind} / \mathrm{m}^{2}\right)$ y Falconidae $\left(0,331 \mathrm{ind} / \mathrm{m}^{2}\right)$. Las familias menos abundantes en esta estación fueron Tytiridae, Icteridae, Furnariidae, Corvidae y Ardeidae, con densidades de $0,025 \mathrm{ind} / \mathrm{m}^{2}$ (figura 4).

Figura 4. Densidad relativa de las familias de aves registradas en la estación 1 y la estación 2 en la Reserva el Caduceo, La María, San Martín (Meta, Colombia)

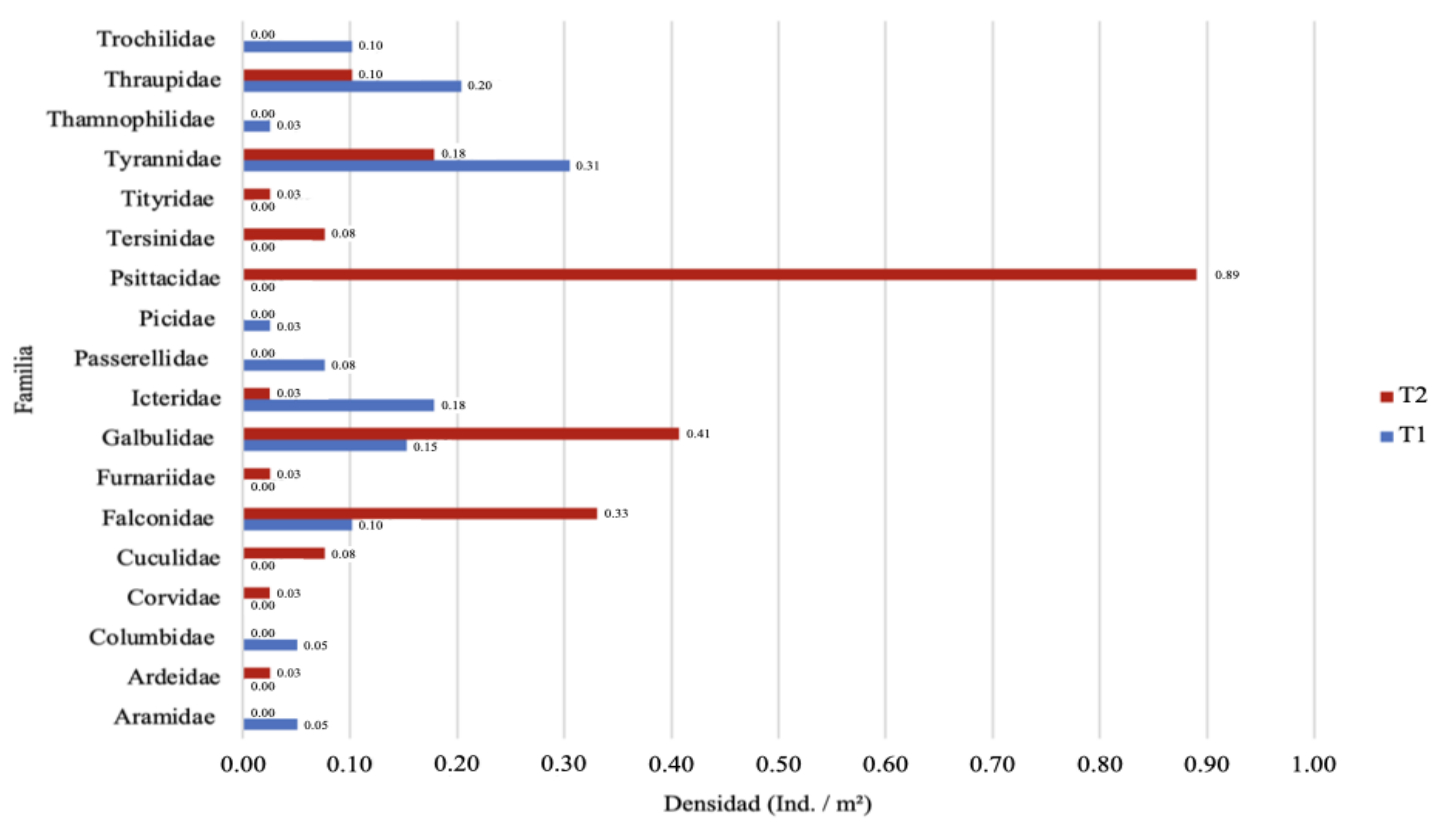

Fuente: elaboración propia.

\section{DISCUSIÓN}

De acuerdo con los rangos propuestos por Ramírez (2006) y con base en el índice de dominancia de Simpson $(\lambda)$, se puede afirmar que las comunidades evaluadas no presentan familias de aves dominantes $(0<\lambda \leq 1)$. El índice de equitatividad de Pielou en T1 muestra que las densidades poblacionales no están bien repartidas entre las 11 familias registradas, mientras que para $\mathrm{T} 2$ las densidades poblacionales se encuentran equitativamente repartidas entre las 12 familias $(0<J \leq 1)$ (Ramírez, 2006). Estas diferencias pueden estar asociadas al tipo de hábitat que predomina en cada estación, dado que se ha visto que a mayor complejidad estructural vegetativa, mayor composición de especies de aves. Adicionalmente, se sabe que los bosques de galería son espacios con mayor estratificación tanto horizontal como vertical, lo cual genera una mayor disponibilidad de hábitats y nichos ecológicos para distintas especies de aves (Ramírez-Albores, 2010).

Las comunidades evaluadas exhiben una similitud muy baja $\left(0<I_{j}<0.5\right)$ (Ramírez, 2006). Esta diferencia en la composición de familias entre las dos estaciones puede deberse al cambio de unidad paisajística, dado que T1 es una zona que ha sido intervenida por actividades de monocultivos, cuya intensificación puede generar un paisaje más abierto $y$, con ello, desencadenar la migración de aves arbóreas 
y la inmigración de aves no arbóreas (Bolwig et al., 2016). Estos hallazgos concuerdan con lo expuesto por Tamaris-Turizo et al. (2017), quienes mencionan que las zonas intervenidas suelen estar dominadas por un número reducido de aves, relegando a aquellas aves asociadas a los bosques, las cuales quedan confinadas en estas zonas, como posiblemente sucede en T2. Esto también puede estar relacionado con la densidad de aves, que fue mayor en T2 con relación a T1, lo que puede soportar que el bosque de galería podría mostrar mayor complejidad estructural, como sostienen algunas investigaciones que sugieren que la abundancia disminuye en zonas donde la complejidad estructural es menor a causa de actividades antropogénicas (Salas-Correa \& ManceraRodríguez, 2018). Así, este bosque de galería, que se encuentra en un proceso de sucesión ecológica (Díaz, 2016; Tamaris-Turizo et al., 2017), puede estar adquiriendo mayor complejidad estructural, lo cual podría ser un indicativo de su recuperación.

Esto último puede estar relacionado con los grupos tróficos que allí predominan, puesto que en esta reserva existen zonas que han sufrido notables modificaciones paisajísticas a causa de diferentes actividades antropogénicas (Huertas-Ramírez \& Huertas-Herrera, 2015; Peñuela et al., 2011; Tamaris-Turizo et al., 2017). Por ello, la predominancia de grupos tróficos como los insectívoros y frugívoros puede permitir la recuperación de la zona y aumentar la complejidad paisajística a través de su capacidad de dispersión de semillas (Herrera, 2004; Stratford \& Pekercioðlu, 2015). Las aves insectívoras, por su parte, influyen en el crecimiento de los árboles al reducir el efecto de herbivoría por parte de los artrópodos folívoros (Stratford \& Pekerciođlu, 2015), por lo que sus preferencias en la dieta contribuyen en el aumento de la complejidad estructural en T2 (Stratford \& Pekercioðlu, 2015; Tamaris-Turizo et al., 2017). Estos grupos tróficos se ven favorecidos durante las épocas de lluvia (tiempo en el que se realizó el muestreo) debido a que hay mayor disponibilidad de agua y, por lo tanto, una mayor estimulación en la producción de hojas y frutos, lo que aumenta la productividad neta del bosque, favoreciendo las comunidades de artropofauna (ClavijoAwazacko \& Amarillo-Suárez, 2013; Stratford \& Pekercioðlu, 2015; Zuluaga, 2017). Esto se traduce en una mayor disponibilidad de recursos alimenticios tanto para aves frugívoras como insectívoras.
Adicionalmente, se resalta que en T1 se evidenció predominancia de las aves insectívoras, lo cual concuerda con estudios previos en zonas de monocultivos, donde esta preferencia dietaria actúa como control de insectos que representan un riesgo para estas actividades (Achondo et al., 2011; Azhar et al., 2013; Enríquez, 2006).

Finalmente, la similitud encontrada entre la presencia de algunas familias y las dos estaciones de muestreo puede deberse a la dependencia de las aves hacia los bosques (Enríquez, 2006), puesto que en ambas estaciones fue posible encontrar bosques de galería; aunque en T1 se observaron pequeños núcleos de bosques aislados. Esto puede estar relacionado con las preferencias de hábitat, dado que el común denominador entre las familias Falconidae, Galbulidae, Icteridae, Thraupidae y Tyrannidae es su preferencia por hábitats de tipo boscoso (Fraga, 2008; Fuchs et al., 2015; Graber et al., 1974; Mason \& Burns, 2015; Pinheiro \& Campos, 2019). Lo anterior sugiere la importancia de mantener fragmentos boscosos en zonas intervenidas, considerando que estos tendrían efectos positivos sobre la avifauna y, por ende, sobre la diversidad de estas áreas.

\section{CONCLUSIÓN}

Este estudio permite una evaluación rápida de la composición a nivel de familia y de grupos tróficos de aves a partir de sus hábitos dietarios, mostrando de forma preliminar la relación entre estos atributos y la complejidad estructural del bosque de galería en recuperación. A pesar de ello, se requieren más estudios que permitan validar esta afirmación y que relacionen de forma directa la diversidad de aves y las preferencias dietarias con el tipo de vegetación, así como la composición de la comunidad vegetal. No obstante, se resalta la importancia de las funciones tróficas que predominan en esta zona, las cuales pueden estar asociadas con el aumento de la complejidad paisajística, especialmente en los núcleos de bosque de galería. Se sugieren futuros estudios de mayor duración y más robustos que permitan validar los hallazgos aquí presentados, así como la realización de evaluaciones del estado de la diversidad en otras zonas de la reserva que puedan estar afectadas por las actividades antropogénicas allí desarrolladas. 


\section{AGRADECIMIENTOS}

Los autores agradecen a la Universidad de Bogotá Jorge Tadeo Lozano y a los programas de Biología Marina y Biología Ambiental, de la Facultad de Ciencias Naturales e Ingenierías, por la oportunidad de desarrollar ejercicios de campo dentro de las asignaturas, los cuales permiten obtener resultados relevantes para su divulgación como ejercicios académicos. También agradecen al doctor Miguel Ángel Suárez, representante legal de La Reserva El Caduceo, quien amablemente prestó apoyo logístico para el trabajo en campo, y al personal de la reserva.

\section{CONSIDERACIONES ÉTICAS}

Los autores declaran que ningún organismo fue capturado ni lastimado durante los muestreos desarrollados.

\section{REFERENCIAS}

Acevedo-Charry, O., Pinto-Gómez, A., \& Rangel-Ch, J. (2014). Las aves de la Orinoquía colombiana: una revisión de sus registros. En J. O. Rangel-Ch (ed.), Colombia. Diversidad Biótica XIV. La región de la Orinoquia de Colombia (pp. 691-750). Universidad Nacional de Colombia \& Instituto de Ciencias Naturales.

Achondo, M., Casin, L., Bello, V., Tanalgo, K., Agduma, A., Bretaña, B., Mancao, L., Salem, J., \& Supremo, J. (2011). Rapid assessment and feeding guilds of birds in selected rubber and oil palm plantations in North Cotabato. Asian Journal of Biodiversity, 2, 103-120. https://doi.org/10.15517/rbt. v65i4.26266

Asociación Colombiana de Ornitología. (2020). Lista de referencia de especies de aves de Colombia - 2020 (V2) [Data set/checklist]. Asociación Colombiana de Ornitología. http://doi. org/10.15472/qhsz0p

Azhar, B., Lindenmayer, D., Wood, J., Fischer, J., Manning, A., McElhinny, C., \& Zakaria, M. (2013). The influence of agricultural system, stand structural complexity and landscape context on foraging birds in oil palm landscapes. Ibis, 155, 297-312. https://doi.org/10.1111/ibi.12025
Bolwig, S., Pomeroy, D., Tushabe, H., \& Mushabe, D. (2006). Crops, trees, and birds: Biodiversity change under agricultural intensification in Uganda's farmed landscapes. Geografisk Tidsskrift, 106, 115-130. https://doi.org/10.1080/ 00167223.2006 .10649561

Cantillo, A. (2009). Diversidad del ensamblaje de renacuajos de un bosque de galería y su relación con las variables ambientales y estructurales en la Reserva El Caduceo, San Martín, Meta (tesis de grado, Pontificia Universidad Javeriana). Repositorio Pontificia Universidad Javeriana.

Casallas-Pabón, D., Calvo-Roa, N., \& Rojas-Robles, R. (2017). Murciélagos dispersores de semillas en gradientes sucesionales de la Orinoquia (San Martín, Meta, Colombia). Acta Biológica Colombiana, 22(3), 348-358. https://doi. org/10.15446/abc.v22n3.63561

Clavijo-Awazacko, H., \& Amarillo-Suárez, Á. (2013). Variación taxonómica y funcional en la artropofauna asociada a comunidades vegetales en humedales altoandinos (Colombia). Revista Colombiana de Entomología, 39(1), 155-163.

Díaz, I. (2016). Paisajes palmeros en los llanos colombianos: estado, nostalgia y trabajo en San Martín, Meta (tesis de maestría, Universidad Nacional de Colombia). Repositorio Institucional Universidad Nacional de Colombia.

Enríquez, L. (2006). Estructura y composición de la comunidad de aves asociadas a plantaciones de palma de aceite (Elaeis guineensis Jacq.) en Agropecuaria La Loma (Acacias - Meta) (tesis de pregrado, Pontificia Universidad Javeriana). Repositorio Pontificia Universidad Javeriana.

Fonseca, A. N., \& Mahecha, O. (2018). Contribución al estudio de los arácnidos (Arachnida) asociados a un bosque de galería en Meta (Colombia). Revista Ibérica de Aracnología, 33(1), 123-128.

Fraga, R. (2008). Phylogeny and behavioral evolution in the family Icteridae. Ornitología Neotropical, 19, 61-71. http://hdl.handle.net/11336/80656

Fuchs, J., Johnson, J. A., \& Mindell, D. P. (2015). Rapid diversification of falcons (Aves: Falconidae) due to expansion of open habitats in the 
Late Miocene. Molecular Phylogenetics and Evolution, 82, 166-182. https://doi.org/10.1016/j. ympev.2014.08.010

Gallina, S., \& López-Gonzáles, C. (2011). Manual de técnicas para el estudio de la fauna (vol. 1). Instituto de Ecología, Universidad Autónoma de Querétaro.

García, S., Flores, R., García, J., \& Salas, J. (2019). Diversidad y estructura vertical del bosque de pino-encino en Guadalupe y Calvo, Chihuahua. Revista Mexicana de Ciencias Forestales, 10(53), 41-63. https://doi.org/10.29298/rmcf.v10i53.173

González-García, F. (2011). Métodos para contar aves terrestres. En S. Gallina (ed.), Manual de técnicas para el estudio de la fauna (pp. 128-147). Inecol.

González-Salazar, C., Martínez-Meyer, E., \& LópezSantiago, G. (2014). Clasificación jerárquica de gremios tróficos para aves y mamíferos de Norteamérica. Revista mexicana de biodiversidad, 85(3), 931-941. http://dx.doi.org/10.7550/ rmb.38023

Google Maps. (2020, octubre 27). Reserva EI Caduceo, Colombia. https://www.google.com/ maps/place/Reserva+El+Caduceo/@3.6667104,$73.6576606,1004$ mdata $=! 3 \mathrm{~m} 1$ ! $1 \mathrm{e} 3 ! 4 \mathrm{~m} 5$ ! $3 \mathrm{~m} 4$ ! $1 \mathrm{~s} 0$ x8e3e08a966517f95:0x9f5470dd11190ad2!8m2! $3 d 3.6653584 ! 4 d-73.6578285$

Graber, J. W., Graber, R. R., \& Kirk, E. L. (1974). Illinois birds: Tyrannidae. Illinois Natural History Survey, Prairie Research Institute, University of Illinois at Urbana-Champaign.

Herrera, C. (2004). Ecología de los pájaros frugívoros ibéricos. En J. L. Tellería (ed.), La hornotología hoy: homenaje al Profesor Francisco Bernis (pp. 127-153). Universidad Complutense de Madrid.

Huertas-Ramírez, H., \& Huertas-Herrera, A. (2015). Historiografía de la ganadería en la Orinoquía. Actas Iberoamericanas de Conservación Animal, 6, 300-307.

León, L., \& Mojica, R. (2018). Géneros de escarabajos coprófagos (scarabaeidae: scarabaeinae) de dos zonas de la reserva natural El Caduceo, San Martín Meta, Colombia (tesis de grado,
Universidad Pedagógica Nacional). Repositorio Universidad Pedagógica Nacional.

MacGregor-Fors, I., Morales-Pérez, L., \& Schondube, J. (2010). Migrating to the city: Responses of neotropical migrant bird communities to urbanization. The Condor, 112, 711-717. https:// doi.org/10.1525/cond.2010.100062

Mason, N., \& Burns, K. (2015). The effect of habitat and body size on the evolution of vocal displays in Thraupidae (tanagers), the largest family of songbirds. Biological Journal of the Linnean Society, 114(3), 538-551. https://doi. org/10.1111/bij.12455

Martínez, O., Olivera, M., Quiroga, C., \& Gómez, I. (2010). Evaluación de la avifauna de la ciudad de La Paz, Bolivia. Revista Peruana de Biología, 17(2), 197-206. https://doi.org/10.15381/rpb.v17i2.28

Melo-Velasquez, D., \& Vega-Araque, D. (2019). San Martín del Meta, Colombia Aves de la Reserva Natural El Caduceo. Universidad Distrital Francisco José de Caldas. https://fieldguides. fieldmuseum.org/sites/default/files/rapidcolor-guides-pdfs/1118_colombia_birds_of_el_ caduceo_natural_reserve.pdf

Moreno, C. (2017). Diversidad taxonómica y funcional de aves asociadas a diferentes tipos de vegetación (tesis de maestría, Pontificia Universidad Javeriana). Repositorio Pontificia Universidad Javeriana. http://hdl.handle. net/10554/34368

Olsen, A. (2017). Feeding ecology is the primary driver of beak shape diversification in waterfowl. Functional Ecology, 31(10), 1985-1995. https:// doi.org/10.1111/1365-2435.12890

Peñuela, L., Castro, F., \& Ocampo-Peñuela, N. (2011). Las reservas naturales del nodo orinoquia en su rol de conservación de la biodiversidad. Fundación Horizonte Verde \& Resnatur.

Pinheiro, C. E. G., \& Campos, V. C. (2019). The responses of wild jacamars (Galbula ruficauda, Galbulidae) to aposematic, aposematic and cryptic, and cryptic butterflies in central Brazil. Ecological Entomology, 44(4), 441-450. https:// doi.org/10.1111/een.12723 
Ralph, J., Geupel, G., Pyle, P., Martin, T., DeSante, D., \& Milá, B. (1996). Manual de métodos de campo para el monitoreo de aves terrestres. U.S. Department of Agriculture. https://doi. org/10.2737/psw-gtr-159

Ramírez, A. (2006). Ecología: Métodos de muestreo y análisis de poblaciones y comunidades. Pontificia Universidad Javeriana.

Ramírez-Albores, J. E. (2010). Diversidad de aves de hábitats naturales y modificados en un paisaje de la Depresión Central de Chiapas, México. Revista de Biología Tropical, 58(1), 511-528. https://doi. org/10.15517/rbt.v58i1.5225

Salas-Correa, Á., \& Mancera-Rodríguez, N. (2018). Relaciones entre la diversidad de aves y la estructura de vegetación en cuatro etapas sucesionales de bosque secundario, Antioquia, Colombia. Revista U.D.C.A Actualidad \& Divulgación Científica, 21(2), 519-529. https://doi. org/10.31910/rudca.v21.n2.2018.970

Stratford, J., \& Pekercioðlu, Ç. (2015). Birds in forest ecosystems. In S. H. Kelvin, R. T. Corlett, \& Y. Bergeron (eds.), Routledge Handbook of Forest Ecology (pp. 279-294). Routledge.

Tamaris-Turizo, D., López-Arévalo, H., \& Romero, N. (2017). Efecto de la estructura del cultivo de palma de aceite Elaeis guineensis (Arecaceae) sobre la diversidad de aves en un paisaje de la Orinoquía colombiana. Revista de Biología Tropical, 65(4), 1569-1581. https://doi. org/10.15517/rbt.v65i4.26735
Taylor, M., Salazar, J., Enríquez, P., León-Cortés, J., \& García-Estrada, C. (2017). Variation in hierarchical guild structure between two bird assemblages of a wetland in the Mexican Pacific. Revista de Biología Tropical, 65(4), 1540-1553. https://doi. org/10.15517/rbt.v65i4.26266

Wikipedia. (2012, noviembre 29). San Martín (Meta). https://es.wikipedia.org/wiki/ San_Mart\%C3\%ADn_(Meta).

Winkler, H., \& Preleuthner, M. (2001). Behaviour and ecology of birds in tropical rain forest canopies. Plant Ecology, 153, 193-202. https://doi. org/10.1007/978-94-017-3606-0_15

Zuluaga, J. (2017). Diversidad composicional y funcional de aves en diferentes tipos de vegetación durante el periodo de lluvias en el enclave seco del Cañón del río Chicamocha, Santander (tesis de maestría, Pontificia Universidad Javeriana). Repositorio Pontificia Universidad Javeriana. 